\title{
Cosmological Big Bounce Transition
}

\author{
Włodzimierz Piechocki \\ Theoretical Physics Department, Institute for Nuclear Studies, Hoża 69, 00-681 Warsaw, Poland; \\ e-mail:piech@fuw.edu.pl
}

\begin{abstract}
We analyze the big bounce transition of the quantum FRW model in the setting of the nonstandard loop quantum cosmology (LQC). Elementary observables are used to quantize compound observables. The spectrum of the energy density operator is bounded and continuous. The spectrum of the volume operator is bounded from below and discrete. It has equally distant levels defining a quantum of the volume. The discreteness may imply a foamy structure of spacetime at semiclassical level which may be detected in astro-cosmo observations. The nonstandard LQC method has a free parameter that should be fixed in some way to specify the big bounce transition.
\end{abstract}

Keywords: Cosmological singularity, nonstandard LQC, spectra of observables

PACS: 98.80.Qc, 04.60.Pp, 04.20.Jb

\section{INTRODUCTION}

An evidence for the existence of the cosmological singularity (diverging gravitational and matter fields invariants) is roughly speaking the following: (i) observational cosmology - our universe emerged from a state with extremely high energy densities of matter fields, and (ii) theoretical cosmology - most of known general relativity (GR) models of the universe (Bianchi, Friedmann, Lemaitre, Kasner, ...) predict an existence of the cosmological singularities. An expectation is that quantization may heal the singularities.

There are two alternative methods of canonical quantization of cosmological models of GR, which make use of the loop geometry:

- standard LQC, i.e. Dirac's method, based on the rule: 'first quantize then impose constraints';

- nonstandard LQC, i.e. reduced phase space (RPS) quantization, based on the rule: 'first solve constraints then quantize'.

The results for flat FRW model with massless scalar field, are roughly speaking, the following:

- standard LQC: classical Big Bang is replaced by quantum Big Bounce due to strong quantum effects at the Planck scale (see, e.g. [1, 2] );

- nonstandard LQC (see, e.g. [3, 4] ]):

- modification of GR by loop geometry is responsible for the resolution of the singularity,

- quantization may lead to discrete spectra of physical observables, which may be confronted with the data of observational cosmology. 
There are many intriguing questions to be answered within quantum cosmology. For instance: What is the energy scale of the Big Bounce? What is the structure of spacetime at semi-classical level (simply connected, foamy or discrete)? What is the origin of tiny fluctuations in the energy density visible in the CMB? How long had the quantum phase lasted? What was before the Big Bounce?

In what follows we present results concerning quantization of the two models:

(i) isotropic and homogeneous universe - flat FRW model with massless scalar field [3, 4], and with cosmological constant $\Lambda[5,6]$, and (ii) homogeneous universe - the Bianchi I model with massless scalar field [7, 8].

\section{CLASSICAL LEVEL}

\section{Modified Hamiltonian}

The gravitational part of of the Hamiltonian of FRW universe with massless scalar field reads [3]

$$
H_{g}=-\gamma^{-2} \int_{\mathscr{V}} d^{3} x e^{-1} \varepsilon_{i j k} E^{a j} E^{b k} F_{a b}^{i}
$$

where $\gamma$, Barbero-Immirzi parameter; $\mathscr{V} \subset \Sigma$, fiducial cell; $\varepsilon_{i j k}$, alternating tensor; $E_{i}^{a}$, density weighted triad; $e:=\sqrt{|\operatorname{det} E|} ; F_{a b}^{k}=\partial_{a} A_{b}^{k}-\partial_{b} A_{a}^{k}+\varepsilon_{i j}^{k} A_{a}^{i} A_{b}^{j}$, curvature of $S U(2)$ connection $A_{a}^{i}$.

By modification we mean the following approximation applied to $F_{a b}^{k}$ :

$$
F_{a b}^{k}(\lambda) \approx-2 \operatorname{Tr}\left(\frac{h_{L_{i j}}^{(\lambda)}-1}{\lambda^{2}}\right) \tau^{k o} \omega_{a}^{i o} \omega_{a}^{j} .
$$

where holonomy of the connection, around the square (loop) $L_{i j}$ with sides length $\lambda$, is $h_{L_{i j}}^{(\lambda)}=h_{i}^{(\lambda)} h_{j}^{(\lambda)}\left(h_{i}^{(\lambda)}\right)^{-1}\left(h_{j}^{(\lambda)}\right)^{-1}$. An exact formula reads

$$
F_{a b}^{k}=\lim _{\lambda \rightarrow 0} F_{a b}^{k}(\lambda)
$$

The holonomy $h_{s}(A)$ of connection $A$ along a curve $s:[0,1] \rightarrow s(t) \in \mathscr{V}$ is the solution to the equation

$$
\frac{d}{d t} h_{s_{t}}(A)=A\left(s_{t}\right) h_{s_{t}}(A), \quad h_{s_{0}}=I
$$

where $A\left(s_{t}\right):=A_{a}^{j}\left(s_{t}\right) \tau_{j} \dot{s}^{a}(t), h_{s}(A):=h_{s_{1}}(A) \in S U(2), s_{t} \equiv s(t)$.

The holonomy along straight edge ${ }^{o} e_{k}^{a} \partial_{a}$ of length $\lambda$ (in fundamental, $\mathrm{j}=1 / 2$, representation of $\mathrm{SU}(2)$ group) reads

$$
h_{k}^{(\lambda)}(c)=\exp \left(\tau_{k} \lambda c\right)=\cos (\lambda c / 2) I+2 \sin (\lambda c / 2) \tau_{k},
$$

where $A_{a}^{k}={ }^{o} \omega_{a}^{k} c$ and $\tau_{k}=-i \sigma_{k} / 2$ ( $\sigma_{k}$ are the Pauli spin matrices). 
The total Hamiltonian for FRW universe with a massless scalar field $\phi$ is given by

$$
H=H_{g}+H_{\phi}, \quad H_{\phi}:=p_{\phi}^{2}|p|^{-\frac{3}{2}} / 2
$$

where $\phi$ and $p_{\phi}$ are elementary variables satisfying $\left\{\phi, p_{\phi}\right\}=1$.

Making use of (5) we calculate (1) and get the modified total Hamiltonian corresponding to (6)

$$
H^{(\lambda)} / N=-\frac{3}{8 \pi G \gamma^{2}} \frac{\sin ^{2}(\lambda \beta)}{\lambda^{2}} v+\frac{p_{\phi}^{2}}{2 v},
$$

where the canonical variables (of the improved scheme) read

$$
\beta:=\frac{c}{|p|^{1 / 2}}, \quad v:=|p|^{3 / 2}
$$

where $\beta \sim \dot{a} / a$ and $v \sim a^{3}$ (for $\lambda=0$ ). Eq (7) presents a modified classical Hamiltonian; it includes no quantum physics.

The Poisson bracket for the canonical variables $\left(\beta, v, \phi, p_{\phi}\right)$ is defined to be

$$
\{\cdot, \cdot\}:=4 \pi G \gamma\left[\frac{\partial \cdot}{\partial \beta} \frac{\partial \cdot}{\partial v}-\frac{\partial \cdot}{\partial v} \frac{\partial \cdot}{\partial \beta}\right]+\frac{\partial \cdot}{\partial \phi} \frac{\partial \cdot}{\partial p_{\phi}}-\frac{\partial \cdot}{\partial p_{\phi}} \frac{\partial \cdot}{\partial \phi} .
$$

The dynamics of a canonical variable $\xi$ is given by Hamilton's equations

$$
\dot{\xi}:=\left\{\xi, H^{(\lambda)}\right\}, \quad \xi \in\left\{\beta, v, \phi, p_{\phi}\right\}
$$

where $\dot{\xi}:=d \xi / d \tau$, and where $\tau$ is an evolution parameter. Dynamics in physical phase space, $\mathscr{F}_{\text {phys }}^{(\lambda)}$, is defined by solutions to $(10)$ satisfying the condition $H^{(\lambda)} \approx 0$. Solutions of (10) ignoring the constraint $H^{(\lambda)} \approx 0$ are in kinematical phase space, $\mathscr{F}_{\text {kin }}^{(\lambda)}$.

\section{Physical phase space}

Equation (7) can be rewritten as

$$
H^{(\lambda)}=N H_{0}^{(\lambda)} \tilde{H}^{(\lambda)} \approx 0
$$

where

$$
H_{0}^{(\lambda)}:=\frac{3}{8 \pi G \gamma^{2} v}\left(\kappa \gamma\left|p_{\phi}\right|+v \frac{|\sin (\lambda \beta)|}{\lambda}\right), \quad \tilde{H}^{(\lambda)}:=\kappa \gamma\left|p_{\phi}\right|-v \frac{|\sin (\lambda \beta)|}{\lambda} .
$$

It is clear that $H_{0}^{(\lambda)}=0$ iff $p_{\phi}=0=\sin (\lambda \beta)$. In such a case $\tilde{H}^{(\lambda)}=0$, thus $H^{(\lambda)}$ equals identically zero so there is no dynamics. We exclude such pathological case from further considerations. Since $N H_{0}^{(\lambda)} \neq 0$, the original dynamics may be reduced to the relative dynamics with the simpler constraint, $\tilde{H}^{(\lambda)} \approx 0$. 
For functions $f$ and $g$ on $\mathscr{F}_{\text {phys }}^{(\lambda)}$ we have

$$
\begin{gathered}
\dot{f}=\left\{f, N H_{0}^{(\lambda)} \tilde{H}^{(\lambda)}\right\}=N H_{0}^{(\lambda)}\left\{f, \tilde{H}^{(\lambda)}\right\}, \\
\dot{g}=\left\{g, N H_{0}^{(\lambda)} \tilde{H}^{(\lambda)}\right\}=N H_{0}^{(\lambda)}\left\{g, \tilde{H}^{(\lambda)}\right\} .
\end{gathered}
$$

The relation

$$
\frac{\dot{f}}{\dot{g}}=\frac{d f}{d g}=\frac{N H_{0}^{(\lambda)}\left\{f, \tilde{H}^{(\lambda)}\right\}}{N H_{0}^{(\lambda)}\left\{g, \tilde{H}^{(\lambda)}\right\}}=\frac{\left\{f, \tilde{H}^{(\lambda)}\right\}}{\left\{g, \tilde{H}^{(\lambda)}\right\}}, \quad \text { as } \quad H_{0}^{(\lambda)} \neq 0
$$

may be rewritten as

$$
\frac{d f}{\left\{f, \tilde{H}^{(\lambda)}\right\}}=\frac{d g}{\left\{g, \tilde{H}^{(\lambda)}\right\}} .
$$

Thus, in the relative dynamics one canonical variable may be used as an 'evolution parameter' if that variable is a monotonic function.

Equations of motion, in the gauge $N=1 / H_{0}^{(\lambda)}$, read

$$
\begin{gathered}
\dot{\phi}=\kappa \gamma \operatorname{sgn}\left(p_{\phi}\right), \quad \dot{p_{\phi}}=0, \\
\dot{\beta}=-4 \pi G \gamma \frac{|\sin (\lambda \beta)|}{\lambda}, \quad \dot{v}=4 \pi G \gamma \nu \cos (\lambda \beta) \operatorname{sgn}(\sin (\lambda \beta)), \\
\tilde{H}^{(\lambda)} \approx 0,
\end{gathered}
$$

where $\kappa^{2}=4 \pi G / 3$. Due to (17), $\phi$ is a monotonic function.

The solution of the relative dynamics is found to be:

$$
v=\frac{\Delta}{2} \cosh \left(3 \kappa s\left(\phi-\phi_{0}\right)-\ln \Delta\right), \quad \beta=\frac{1}{\lambda} \arcsin (\Delta / v),
$$

where $\Delta:=\kappa \gamma \lambda\left|p_{\phi}\right|$ and $s:=\operatorname{sgn}\left(p_{\phi}\right)$. The variables $v$ and $\beta$ are functions of the evolution parameter $\phi$.

\section{Elementary observables}

A function, $\mathscr{O}: \mathscr{F}_{\text {kin }}^{(\lambda)} \rightarrow R$, is a Dirac observable if

$$
\left\{\mathscr{O}, H^{(\lambda)}\right\}=0
$$

Thus, $\mathscr{O}$ is solution to the equation

$$
\frac{\sin (\lambda \beta)}{\lambda} \frac{\partial \mathscr{O}}{\partial \beta}-v \cos (\lambda \beta) \frac{\partial \mathscr{O}}{\partial v}-\frac{\kappa \gamma \operatorname{sgn}\left(p_{\phi}\right)}{4 \pi G} \frac{\partial \mathscr{O}}{\partial \phi}=0
$$


The simplest solutions to (22), which we call elementary observables, are found to be

$$
\mathscr{O}_{1}:=p_{\phi}, \quad \mathscr{O}_{2}:=\phi-\frac{\operatorname{sgn}\left(p_{\phi}\right)}{3 \kappa} \operatorname{arth}(\cos (\lambda \beta)), \quad \mathscr{O}_{3}:=\operatorname{sgn}\left(p_{\phi}\right) \nu \frac{\sin (\lambda \beta)}{\lambda} .
$$

One may easily verify that the elementary observables satisfy the Lie algebra

$$
\left\{\mathscr{O}_{2}, \mathscr{O}_{1}\right\}=1, \quad\left\{\mathscr{O}_{1}, \mathscr{O}_{3}\right\}=0, \quad\left\{\mathscr{O}_{2}, \mathscr{O}_{3}\right\}=\gamma \kappa .
$$

Due to the constraint $\tilde{H}^{(\lambda)}=0$, we have

$$
\mathscr{O}_{3}=\gamma \kappa \mathscr{O}_{1} \text {. }
$$

One can show that in the physical phase space, $\mathscr{F}_{\text {phys }}^{(\lambda)}$, we have only two elementary observables which satisfy the algebra

$$
\left\{\mathscr{O}_{2}, \mathscr{O}_{1}\right\}=1
$$

where

$$
\{\cdot, \cdot\}:=\frac{\partial \cdot}{\partial \mathscr{O}_{2}} \frac{\partial \cdot}{\partial \mathscr{O}_{1}}-\frac{\partial \cdot}{\partial \mathscr{O}_{1}} \frac{\partial \cdot}{\partial \mathscr{O}_{2}} .
$$

The space $\mathscr{F}_{\text {kin }}^{(\lambda)}$ is four dimensional. In relative dynamics one variable is used to parametrize three others. Since the constraint relates two variables, we have only two independent variables.

\section{Compoud observables}

By compound observables we mean functions on phase space which can be expressed in terms of elementary observables and an evolution parameter $\phi$, so they are not observables. However, they do become observables for each fixed value of $\phi$, since in such a case they are only functions of elementary observables.

In what follows we consider two compound observables: the energy density of matter field

$$
\rho(\phi, \lambda)=\frac{1}{2} \frac{1}{(\kappa \gamma \lambda)^{2} \cosh ^{2} 3 \kappa\left(\phi-\mathscr{O}_{2}\right)},
$$

and the volume operator

$$
v(\phi, \lambda)=\kappa \gamma \lambda\left|\mathscr{O}_{1}\right| \cosh 3 \kappa\left(\phi-\mathscr{O}_{2}\right) .
$$

\section{QUANTUM LEVEL}

\section{Energy density operator}

In the Schrödinger representation we have [4]

$$
\mathscr{O}_{1} \rightarrow \widehat{\mathscr{O}}_{1}:=\widehat{x}:=x, \quad \mathscr{O}_{2} \rightarrow \widehat{\mathscr{O}}_{2}:=-i \hbar \partial_{x},
$$


so the energy density operator reads

$$
\widehat{\rho}:=\frac{1}{2(\kappa \gamma \lambda)^{2} \cosh ^{2} 3 \kappa\left(\phi+i \hbar \partial_{x}\right)} .
$$

Solution to the eigenvalue problem

$$
\widehat{\rho} f_{p}=\rho(p) f_{p}
$$

reads

$$
f_{p}(x)=(2 \pi)^{-1 / 2} \exp (\operatorname{ixp} / \hbar), \quad \rho(p)=\frac{1}{2} \frac{1}{(\kappa \gamma \lambda)^{2} \cosh ^{2} 3 \kappa(\phi-p)},
$$

where $p \in R$. So the energy density has the same functional form as the classical one.

The density $\rho$ has maximum at the minimum of $v$ :

$$
\rho_{\max }=\frac{1}{2 \kappa^{2} \gamma^{2}} \frac{1}{\lambda^{2}}
$$

For the Planck scale, substituting $\lambda=l_{P l}$ into (34) gives $\rho_{\max } / \rho_{P l} \simeq 2,07$; for $\rho_{\max }=\rho_{P l}$ we get $\lambda \simeq 1,44 l_{P l}$. Thus, Eg. (34) fits the Planck scale.

The spectrum $\left(0, \frac{1}{2(\kappa \gamma \lambda)^{2}}\right)$ is continuous and bounded for $\lambda \neq 0$. For $\lambda \rightarrow 0$ we get the classical FRW singularity. Since $\lambda$ is a free parameter, finding the critical density of matter corresponding to the Big Bounce is an open problem.

\section{Volume operator}

The classical volume operator, $v$, can be presented as

$$
v=|w|, \quad w:=\kappa \gamma \lambda \mathscr{O}_{1} \cosh 3 \kappa\left(\phi-\mathscr{O}_{2}\right) .
$$

For $\mathscr{O}_{1}$ and $\mathscr{O}_{2}$ we use the Schrödinger representation:

$$
\mathscr{O}_{1} \longrightarrow \widehat{\mathscr{O}}_{1} f(x):=-i \hbar \partial_{x} f(x), \quad \mathscr{O}_{2} \longrightarrow \widehat{\mathscr{O}}_{2} f(x):=\widehat{x} f(x):=x f(x) .
$$

Thus, an explicit form of $\hat{w}$ is given by

$$
\hat{w}=i \frac{\kappa \gamma \lambda \hbar}{2}\left(2 \cosh 3 \kappa(\phi-x) \frac{d}{d x}-3 \kappa \sinh 3 \kappa(\phi-x)\right) .
$$

Solution to the eigenvalue problem is found to be:

$$
\begin{gathered}
\hat{w} f_{a}(x)=a f_{a}(x), \quad a \in R \\
f_{a}(x):=\frac{\sqrt{\frac{3 \kappa}{\pi}} \exp \left(i \frac{2 a}{3 \kappa^{2} \gamma \lambda \hbar} \arctan e^{3 \kappa(\phi-x)}\right)}{\cosh ^{\frac{1}{2}} 3 \kappa(\phi-x)},
\end{gathered}
$$




$$
a=b+6 \kappa^{2} \gamma \lambda \hbar m=b+8 \pi G \gamma \lambda \hbar m,
$$

where $b \in R$ and $m \in Z$. Completion of the span of

$$
\mathscr{F}_{b}:=\left\{f_{a} \mid a=b+8 \pi G \gamma \lambda \hbar m\right\} \subset L^{2}(R),
$$

in the norm of $L^{2}(R)$ leads to $L^{2}(R), \forall b \in R$. The operator $\hat{w}$ is essentially self-adjoint on each span of $\mathscr{F}_{b}$.

Due to the the relation (35), and the spectral theorem on self-adjoint operators, we get the solution of the eigenvalue of the volume operator:

$$
v=|w| \quad \longrightarrow \quad \hat{v} f_{a}:=|a| f_{a} .
$$

The spectrum is bounded from below and discrete. There exists the minimum gap $\triangle:=8 \pi G \gamma \hbar \lambda$ in the spectrum, which defines a quantum of the volume. In the limit $\lambda \rightarrow 0$, corresponding to the classical FRW model, there is no quantum of the volume.

\section{EXTENSION OF RESULTS}

\section{FRW model with cosmological constant}

The Hamiltonian of the flat FRW model with a free massless scalar field and the cosmological constant $\Lambda>0$ has the form [5, 6]:

$$
H^{(\lambda)}=-\frac{3}{8 \pi G \gamma^{2}} \frac{\sin ^{2}(\lambda \beta)}{\lambda^{2}} v+\frac{p_{\phi}^{2}}{2 v}+v \frac{\Lambda}{8 \pi G} .
$$

The eigenvalue problem for the volume operator has the solution

$$
\begin{gathered}
\hat{v} \psi_{b}=|b| \psi_{b}, \quad \psi_{b} \in L^{2}(R), \\
b=a+\frac{4 \pi^{2} G \hbar \gamma \lambda m}{\arctan \sqrt{1 / \delta-1}}, \quad m=0,1,2, \ldots
\end{gathered}
$$

where $0 \neq a \in R$ and $\delta:=\Lambda \gamma^{2} \lambda^{2} / 3$. The spectrum is discrete.

\section{Bianchi I model}

In the case of the Bianchi I model with free massless scalar field we have [7]

$$
H^{(\lambda)}=-\frac{1}{8 \pi G \gamma^{2} \lambda^{2}}\left[\left|p_{1} p_{2}\right|^{3 / 2} \sin \left(c_{1} \mu_{1}\right) \sin \left(c_{2} \mu_{2}\right)+\text { cyclic }\right]+\frac{p_{\phi}^{2}}{2},
$$

where $\mu_{k}:=\sqrt{\lambda /\left|p_{k}\right|}$, for $k=1,2,3$. 
The solution to the eigenvalue problem for the directional $k$-th volume operator $(\mathrm{k}=$ 1,2,3), for the Kasner-unlike case, reads [8]

$$
\hat{v}_{\alpha} \psi_{\alpha}=\alpha \psi_{\alpha}, \quad \psi_{\alpha} \in L^{2}([0, \pi / b]), \quad \alpha=a b, 2 a b, 3 a b, \ldots
$$

where $a=\kappa \gamma \lambda, b=3 \kappa, a b=4 \pi G \gamma \lambda \hbar$.

In the Kasner-like case, two directional volume operators $w_{2}$ and $w_{3}$ have the spectra similar to the Kasner-unlike case. The volume $w_{1}$ has the spectrum similar to the spectrum of a harmonic oscilator in a box [8].

In all cases the spectrum is discrete and does not include the zero volume eigenvalue.

\section{SUMMARY}

Conclusions:

- Modification of classical Hamiltonian, realized by making use of the loop geometry, turns big bang into big bounce (BB).

- No specific $0<\lambda \in R \Rightarrow$ no specific energy density at $\mathrm{BB}$

- BB may occur at any low density, e.g. at the density of water - disagreement with observations;

- BB may occur at any high density, e.g. at $10^{500}$ of the Planck density - conflict with common believe;

- assuming $\lambda=l_{P l}$ leads to $\rho_{\max } / \rho_{P l} \simeq 2,07$, so our model fits the Planck scale.

- Discreteness of the spectra of the volume operators may favor a foamy structure of space at short distances that may be detected in astro-cosmo observations.

Determination of the free parameter $\lambda$ :

- Loop Quantum Cosmology

- FRW model: no $\lambda$ is privileged,

- Bianchi I model: no $\lambda$ is privileged.

- Observational Cosmology

- cosmic photons: no dispersion of photons [9] up to the energy $5 \times 10^{17} \mathrm{GeV}$,

- detection of primordial gravity waves: we hope that imprints of tensor modes on CMB spectrum [10] may be used to determine $\lambda$.

\section{CHALLENGE}

A great challenge is quantization of the Belinskii-Khalatnikov-Lifshitz scenario (see [11] and references therein):

- general solution of GR (corresponding to a non-zero measure subset of all initial conditions);

- presents an evolution of spacetime near the space-like singularity (with diverging gravitational and matter fields invariants); 
- singular solution applies both to the future singularity (Big Crunch) and past singularity (Big Bang);

- BKL scheme appears in the low energy limit of superstring models (with KacMoody algebras as an underlying mathematics);

- unclear if loop LQG/LQC can be used to resolve the singularity problem of BKL.

The quantum BKL scenario (after being constructed) may give the answer to the questions mentioned in the Introduction.

\section{ACKNOWLEDGMENTS}

The author would like to thank the organizers for inspiring atmosphere at the Meeting.

\section{REFERENCES}

1. A. Ashtekar, T. Pawlowski and P. Singh, "Quantum nature of the big bang", Phys. Rev. Lett. 96 (2006) 141301 [arXiv:gr-qc/0602086].

2. A. Ashtekar, T. Pawłowski and P. Singh, "Quantum nature of the big bang: Improved dynamics", Phys. Rev. D 74 (2006) 084003 [arXiv:gr-qc/0607039].

3. P. Dzierzak, P. Malkiewicz and W. Piechocki, "Turning big bang into big bounce: I. Classical dynamics," Phys. Rev. D 80, 104001 (2009) [arXiv:0907.3436].

4. P. Malkiewicz and W. Piechocki, "Turning big bang into big bounce: II. Quantum dynamics," Class. Quant. Grav. 27 (2010) 225018 [arXiv:0908.4029 [gr-qc]].

5. J. Mielczarek and W. Piechocki, "Observables for FRW model with cosmological constant in the framework of loop cosmology," Phys. Rev. D 82 (2010) 043529 [arXiv:1001.3999 [gr-qc]].

6. J. Mielczarek and W. Piechocki, "Quantum of volume in de Sitter space," arXiv:1011.3418 [gr-qc].

7. P. Dzierzak and W. Piechocki, "Bianchi I model in terms of nonstandard loop quantum cosmology: Classical dynamics, ” Phys. Rev. D 80, 124033 (2009) [arXiv:0909.4211 [gr-qc]].

8. P. Malkiewicz, W. Piechocki and P. Dzierzak, "Bianchi I model in terms of nonstandard loop quantum cosmology: Quantum dynamics," arXiv:1010.2930 [gr-qc].

9. F. Aharonian et al., "Limits on an Energy Dependence of the Speed of Light from a Flare of the Active Galaxy PKS 2155-304", Phys. Rev. Lett. 101, 170402 (2008).

10. J. Mielczarek, "Gravitational waves from the Big Bounce," JCAP 0811 (2008) 011 [arXiv:0807.0712 [gr-qc]].

11. V. Belinski, “Cosmological singularity,” AIP Conf. Proc. 1205 (2009) 17 [arXiv:0910.0374 [gr-qc]]. 